\title{
MODELAGEM DA EMISSÃO DE NITROGÊNIO EM DIFERENTES INDICES DE DESENVOLVIMENTO
}

\author{
Carolina Franchini ${ }^{1}$
}

Gilmar de Almeida Gomes ${ }^{2}$

\begin{abstract}
RESUMO
O crescimento populacional e o incremento na renda per capita, estão gerando preocupações sobre como garantir formas mais sustentáveis de desenvolvimento humano (INTEGRATED MODELLING OF GLOBAL ENVIRONMENTAL CHANGE, 2006). O nitrogênio é um indicador deste impacto, por exemplo na forma de óxido de dinitrogênio $\left(\mathrm{N}_{2} \mathrm{O}\right)$ é altamente nocivo ao meio ambiente, pois provoca a chuva ácida e a destruição da camada de ozônio, além de contribuir para o aquecimento global, sendo mais impactante que $\mathrm{o} \mathrm{CO}_{2}$. O aumento industrial e a atividade humana tem acelerado o ciclo do nitrogênio, aumentando sua fixação natural. Este trabalho visa estudar, emissão do nitrogênio em função de diferentes índices econômicos e sociais.
\end{abstract}

PALAVRAS-CHAVE: Nitrogênio. Produção de alimentos. Óxido de dinitrogênio.

\section{MODELING THE IMPACT OF NITROGEN IN FOOD PRODUCTION}

\begin{abstract}
The population growth and the increase in per capita income, are raising concerns about how to ensure more sustainable forms of human development (INTEGRATED MODELLING OF GLOBAL ENVIRONMENTAL CHANGE, 2006). Nitrogen is an indicator of this impact, for example in the form of dinitrogen oxide (N2O) is highly harmful to the environment because it causes acid rain and the destruction of the ozone la yer and contribute to global warming, more impactful that CO2. The increasing industrial and human activity has accelerated the nitrogen cycle, increasing its natural setting. This work aims to study the nitrogen cycle and assess the impact on rural activity and compare with industrial activity.

KEY-WORDS: Nitrogen. Food production. Dinitrogen oxide.

\footnotetext{
${ }_{1}^{1}$ Acadêmica de Engenharia de Alimentos, Universidade do Estado de Santa Catarina. carol_slo@hotmail.com.

${ }^{2}$ Doutor em Química, Professor da Universidade do Estado de Santa Catarina. gilmargomess@yahoo.com.br
} 


\section{MODELADO DEL IMPACTO DE NITRÓGENO EN LA PRODUCCIÓN DE ALIMENTOS}

\section{RESUMEN}

El crecimiento de la población y el aumento del ingreso per cápita, están elevando las preocupaciones sobre la forma de garantizar formas más sostenibles de desarrollo humano (INTEGRADO DE MODELAJE Cambio Ambiental Global, 2006). El nitrógeno es un indicador de este impacto, por ejemplo, en forma de óxido nitroso (N2O) es altamente perjudicial para el medio ambiente, ya que causa la lluvia ácida y la destrucción de la capa de ozono y contribuyen al calentamiento global, más impactante que el CO2. La actividad industrial y humana en aumento se ha acelerado el ciclo del nitrógeno, el aumento de su entorno natural. Este trabajo tiene como objetivo estudiar el ciclo del nitrógeno y evaluar el impacto en la actividad rural y comparar con la actividad industrial.

PALABRAS-CLAVE Nitrógeno. La producción de alimentos. Óxido de dinitrógeno.

\section{INTRODUÇÃO}

A atividade humana tem acelerado o ciclo de nitrogênio terrestre, aumentando a sua fixação natural. Fixação de nitrogênio é a transformação de dinitrogênio $\left(N_{2}\right)$ atmosférico muito abundante, mas biologicamente indisponível, em formas de nitrogênio reduzidas e oxidadas, como amônia $\left(\mathrm{NH}_{3}\right)$, nitrato $\left(\mathrm{NO}_{3}\right)$, óxido de dinitrogênio $\left(\mathrm{N}_{2} \mathrm{O}\right)$ e ácido nítrico (NO) (INTEGRATED MODELLING OF GLOBAL ENVIRONMENTAL CHANGE, 2006).

O nitrogênio é um elemento essencial à vida e ao meio ambiente, porém em grandes quantidades torna-se prejudicial. Seu aumento se dá pelo crescimento das atividades industriais, queima de combustíveis fósseis e a produção agrícola (HYPESCIENCE, 2010). Esse aumento provoca poluição do ar, contaminação das águas e mudanças climáticas.

O depósito de nitrogênio na atmosfera terrestre vem aumentando constantemente com o passar dos anos. Este depósito aumentou três vezes nos tempos pré-industriais e, de lá para cá, já é dez vezes maior (GALLOWAY, et al., 2004).

As ações antropogênicas têm acelerado as deposições no ciclo do nitrogênio. Isso se dá, por exemplo, pelo fato da modernização agrícola, pois as máquinas utilizadas funcionam a base de combustíveis que quando queimados liberam gases e entre eles, o nitrogênio. $O$ uso de fertilizantes também influenciou no aumento de 
nitrogênio atmosférico. Na área urbana os automóveis e as indústrias são as principais poluentes. $O$ uso de fertilizantes à base de nitrogênio cresceu $800 \%$ de 1960 a 2000 (HYPESCIENCE, 2010).

$\mathrm{Na}$ agricultura, bactérias que vivem em relação simbiótica com as plantas são responsáveis pela fixação de nitrogênio em suas raízes. Outro fator agrícola é a utilização incorreta e intensa de fertilizantes, aumentando, significativamente, a quantidade de nitrogênio no solo. O excesso da aplicação desses fertilizantes, não é absorvido, ficando livre no solo e sendo lixiviado para rios e lençóis freáticos, causando eutrofização

Hoje, para aumentar a produção, os fertilizantes são utilizados em larga escala na agricultura. Nitrogênio, fósforo e potássio são os elementos mais comumente encontrados nos fertilizantes industriais. Com o aumento da mecanização agrícola, há, cada vez mais, um esgotamento de nutrientes no solo, assim é necessário, também, o uso de fertilizantes sintéticos para garantir a produtividade, muitas vezes seu uso se dá em excesso.

Eutrofização é um processo gerado pelo excesso de nutrientes nos recursos hídricos causando o esgotamento do oxigênio e podendo levar a morte de animais aquáticos (HYPESCIENCE, 2010). No ecossistema terrestre a eutrofização causada pelo depósito de nitrogênio tem um número significativo de impactos negativos, incluindo a perda da biodiversidade (BOBBINK, et al., 1998).

A contribuição das indústrias no aumento da emissão de nitrogênio é resultante da queima de combustíveis fósseis e biomassa. Com o aumento da industrialização e da frota de veículos, as emissões de nitrogênio aumentaram e mudanças significativas podem ser notadas, como a alteração do ciclo natural do nitrogênio.

As emissões de nitrogênio chegam a 10 milhões de toneladas por ano, provenientes de fontes naturais e 40 milhões de toneladas por ano de fontes antropogênicas: combustão e emissões automobilísticas (CÓNSUL, et al., 2004).

Além disso, o óxido de dinitrogênio $\left(\mathrm{N}_{2} \mathrm{O}\right)$ tem um potencial de aquecimento global de até 237 vezes mais que o dióxido de carbono $\left(\mathrm{CO}_{2}\right)$ por molécula 
(HYPESCIENCE, 2010), porém o segundo ainda é mais liberado que o primeiro, mas a crescente emissão de nitrogênio vem preocupando pela sua nocividade.

Avaliando esses dados, nota-se que o aumento das emissões de nitrogênio na atmosfera terrestre vem trazendo um quadro de risco aos seres humanos por destruir a camada de ozônio, que é responsável pela proteção contra os raios ultravioleta, chuva ácida, entre outros.

Assumindo que o método de determinação da quantidade de nitrogênio adotado pelo (INTEGRATED MODELLING OF GLOBAL ENVIRONMENTAL CHANGE, 2006) esteja correto, para avaliar emissão de nitrogênio em função do desenvolvimento econômico, torna-se necessário utilizar diferentes indicadores econômico. IDH, PIB, etc, para uma melhor compreensão do impacto deste gás.

\section{2- OBJETIVO}

O objetivo do estudo é a quantificar a emissões de nitrogênio nas atividades humanas, como produção de alimentos, industrialização e transportes. Considerando também, a fixação natural de nitrogênio realizada através de bactérias e plantas. Também visa determinar a emissão em função dos diferentes índices de desenvolvimentos.

\section{METODOLOGIA}

Foram utilizados dados da literatura do PIB e IDH para modelar a emissão de nitrogênio em diferentes índices de desenvolvimentos.

Em trabalhos publicados pelo Painel Intergovernamental de Mudanças Climáticas (IPCC, Intergovernmental Panel on Climate Change), a emissão de nitrogênio, Nem, é baseada no Produto Interno Bruto (PIB), ou seja, a partir do desenvolvimento econômico do local.

A primeira etapa deste projeto foi avaliar a emissão de nitrogênio em diferentes estados brasileiros. A segunda etapa é comparar diferentes indicadores de desenvolvimento econômico como Produto Interno Bruto, PIB e Índice de 
Desenvolvimento Humano (IDH). O PIB foi aplicado na equação sugerida por Van Drecht et.al.(2003) no livro Integrated Modelling of Global Environmental Change (IMAGE). A seguir apresenta-se a equação:

$$
\text { 1. } N e m=8+11\left(P I B \div 1,248 \times 10^{12}\right)^{0.5}
$$

Onde:

- Nem: Emissões de nitrogênio per capita diárias;

- PIB: Produto interno bruto (PIB);

- $1,248 \times 10^{12}$ : maior PIB brasileiro de 2010.

Foi utilizado o programa Origin 6.0 para o tratamento dos dados experimentais.

\section{RESULTADOS E DISCUSSÕES}

Utilizando a equação 01 e os valores de PIB, IDH obteve-se os seguintes resultados de emissões de nitrogênio, para cada estado:

Tabela 01: Emissão de nitrogênio por estado relacionado com o PIB de São Paulo de 2010.

\begin{tabular}{|c|c|c|c|l|}
\hline Estado & $\begin{array}{c}\text { PIB 2010 } \\
\text { (bilhões R\$) }\end{array}$ & IDH 2010 & $\begin{array}{c}\text { Emissões SP } \\
\text { (por pessoa por } \\
\text { dia) }\end{array}$ & $\begin{array}{c}\text { População em } \\
\text { 2010 }\end{array}$ \\
\hline São Paulo & 1.248 & 0,783 & $19,00 \mathrm{~g}$ & 41.252 .160 \\
\hline $\begin{array}{c}\text { Rio de } \\
\text { Janeiro }\end{array}$ & 407,0 & 0,761 & $14,30 \mathrm{~g}$ & 15.993 .583 \\
\hline $\begin{array}{c}\text { Minas } \\
\text { Gerais }\end{array}$ & 351,0 & 0,731 & $13,83 \mathrm{~g}$ & 19.595 .309 \\
\hline $\begin{array}{c}\text { Rio Grande } \\
\text { do Sul }\end{array}$ & 252,2 & 0,746 & $12,94 \mathrm{~g}$ & 10.695 .532 \\
\hline Paraná & 217,0 & 0,749 & $12,59 \mathrm{~g}$ & 10.439 .601 \\
\hline Bahia & 154,0 & 0,66 & $11,86 \mathrm{~g}$ & 14.021 .432 \\
\hline $\begin{array}{c}\text { Santa } \\
\text { Catarina }\end{array}$ & 152,2 & 0,774 & $11,84 \mathrm{~g}$ & 6.249 .682 \\
\hline $\begin{array}{c}\text { Distrito } \\
\text { Federal }\end{array}$ & 150,0 & 0,824 & $11,81 \mathrm{~g}$ & 2.562 .963 \\
\hline Goiás & 97,6 & 0,735 & $11,08 \mathrm{~g}$ & 6.004 .045 \\
\hline Pernambuco & 95,0 & 0,673 & $11,03 \mathrm{~g}$ & 8.796 .032 \\
\hline
\end{tabular}




\begin{tabular}{|c|c|c|c|c|}
\hline $\begin{array}{l}\text { Espírito } \\
\text { Santo }\end{array}$ & 82,0 & 0,740 & $10,82 g$ & 3.512 .672 \\
\hline Ceará & 77,9 & 0,682 & $10,75 \mathrm{~g}$ & 8.448 .055 \\
\hline Pará & 77,9 & 0,646 & $10,75 \mathrm{~g}$ & 7.588 .078 \\
\hline Amazonas & 59,8 & 0,674 & $10,41 \mathrm{~g}$ & 3.480 .937 \\
\hline Mato Grosso & 59,6 & 0,725 & $10,41 \mathrm{~g}$ & 3.033 .991 \\
\hline Maranhão & 45,0 & 0,639 & $10,09 \mathrm{~g}$ & 6.569 .683 \\
\hline $\begin{array}{c}\text { Mato Grosso } \\
\text { do Sul }\end{array}$ & 43,5 & 0,729 & $10,05 \mathrm{~g}$ & 2.449 .341 \\
\hline $\begin{array}{c}\text { Rio Grande } \\
\text { do Norte }\end{array}$ & 32,0 & 0,684 & $9,76 \mathrm{~g}$ & 3.168 .133 \\
\hline Paraíba & 31,9 & 0,658 & $9,76 \mathrm{~g}$ & 3.766 .834 \\
\hline Alagoas & 24,6 & 0,631 & $9,54 \mathrm{~g}$ & 3.120 .922 \\
\hline Sergipe & 23,9 & 0,665 & $9,52 \mathrm{~g}$ & 2.068 .031 \\
\hline Rondônia & 23,5 & 0,690 & $9,51 \mathrm{~g}$ & 1.560 .501 \\
\hline Piauí & 22,0 & 0,646 & $9,46 \mathrm{~g}$ & 3.119 .015 \\
\hline Tocantins & 17 & 0,699 & $9,28 \mathrm{~g}$ & 1.388 .455 \\
\hline Acre & 8,5 & 0,663 & $8,91 \mathrm{~g}$ & 732.793 \\
\hline Amapá & 8,3 & 0,708 & $8,90 \mathrm{~g}$ & 668.689 \\
\hline Roraima & 6,3 & 0,707 & $8,80 \mathrm{~g}$ & 451.227 \\
\hline
\end{tabular}

Fonte: IBGE, 2010.

Gráfico 01: PIB de São Paulo relacionado com as emissões de nitrogênio por estado.

Para uma melhor compreensão dos resultados e entender as relações entres eles foram construídos, a partir da Tabela 1, três gráficos e modelado a melhor função matemática que o representa-o, 1- Nem em função do PIB, 2- Nem em função do IDH e 3- Nem em função da população, apresentados abaixo. IM 


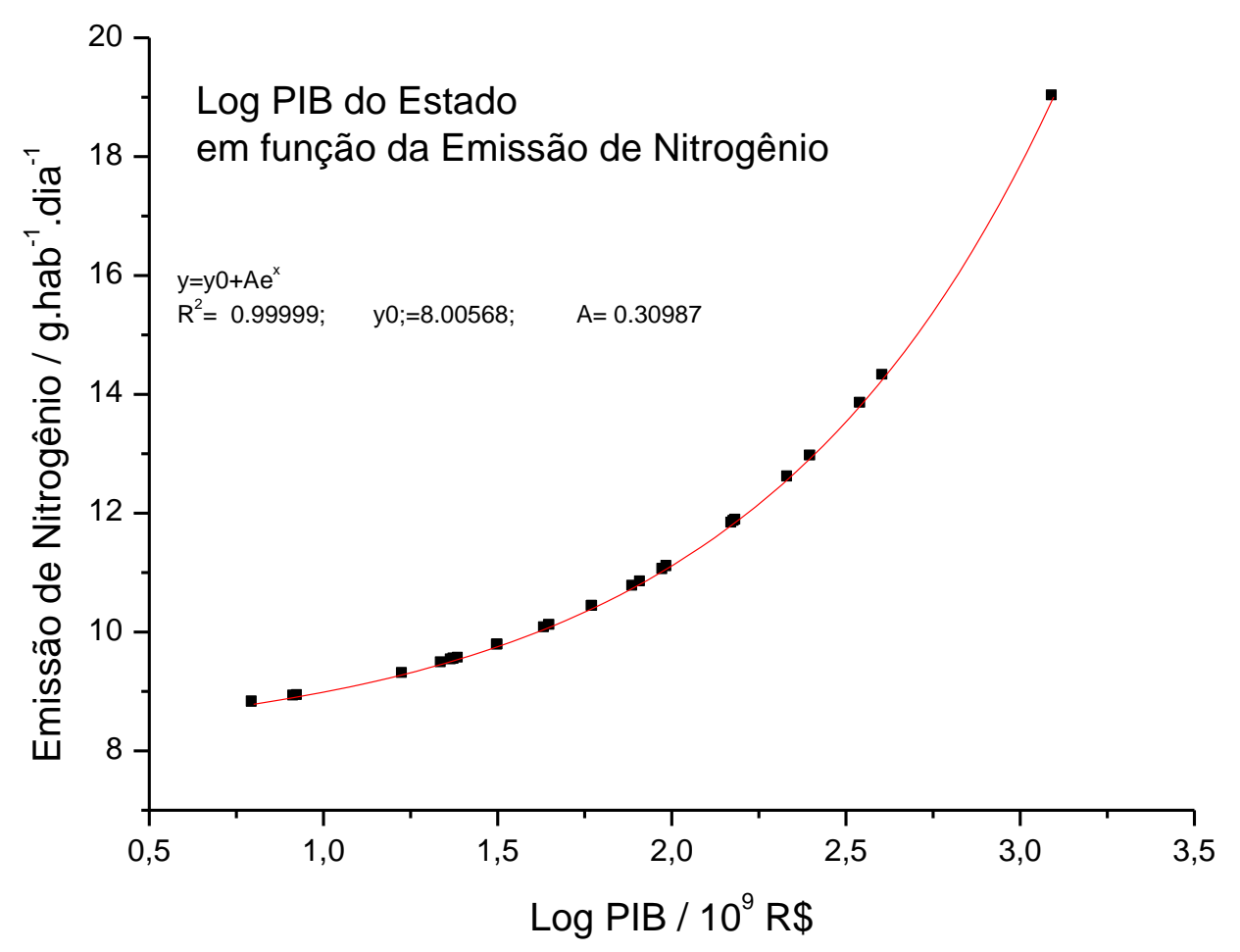

Figura 1 Emissão de nitrogênio em função do log do PIB dos estados brasileiros

Observa-se a partir da Figura 1 que emissão de nitrogênio cresce exponencialmente com o PIB, e uma correlação dos valores calculados muito boa com $R^{2}$ muito próximo de 1 . Também mostra este gráfico que o aumento da economia, medida pelo aumento do PIB, tem como consequência um aumento exponencial no impacto ambiental através da emissão de nitrogênio.

O PIB leva em conta a produção de riquezas de um determinado local, por isso, pode-se ver que o estado de São Paulo, por sua extensão e por sua contribuição no PIB nacional é quem mais libera nitrogênio na atmosfera. Em contrapartida, estados de menor expressão contribuem com menores valores.

Considerando o PIB brasileiro de 2012 como 2,253 trilhões de dólares e o PIB dos Estados Unidos da América como o maior deste Na literatura tem-se que países mais desenvolvidos emitem de 15 a 18 gramas por pessoa por dia e os menos 
desenvolvidos 10 gramas. O que confirma o Brasil como um país em desenvolvimento. Utilizando o total de 15,680 trilhões de dólares, e a equação anterior (eq. 01), obtém-se o valor de 12,2 gramas por pessoa por dia.

Em 1990 o PIB brasileiro era de 462 bilhões de reais, sendo assim, neste ano a emissão de nitrogênio foi de, aproximadamente $10 \mathrm{~g}$ por pessoa por dia. Assim em, aproximadamente 2 décadas, a emissão aumentou em 2 gramas, o que pode ser explicado pelo avanço das indústrias e a modernização da agricultura.

Utilizando a equação 1 e investigando outros índices de desenvolvimento, diferente do PIB, foi calculado a emissão de nitrogênio em função do IDH, apresentado na Figura 2 abaixo.

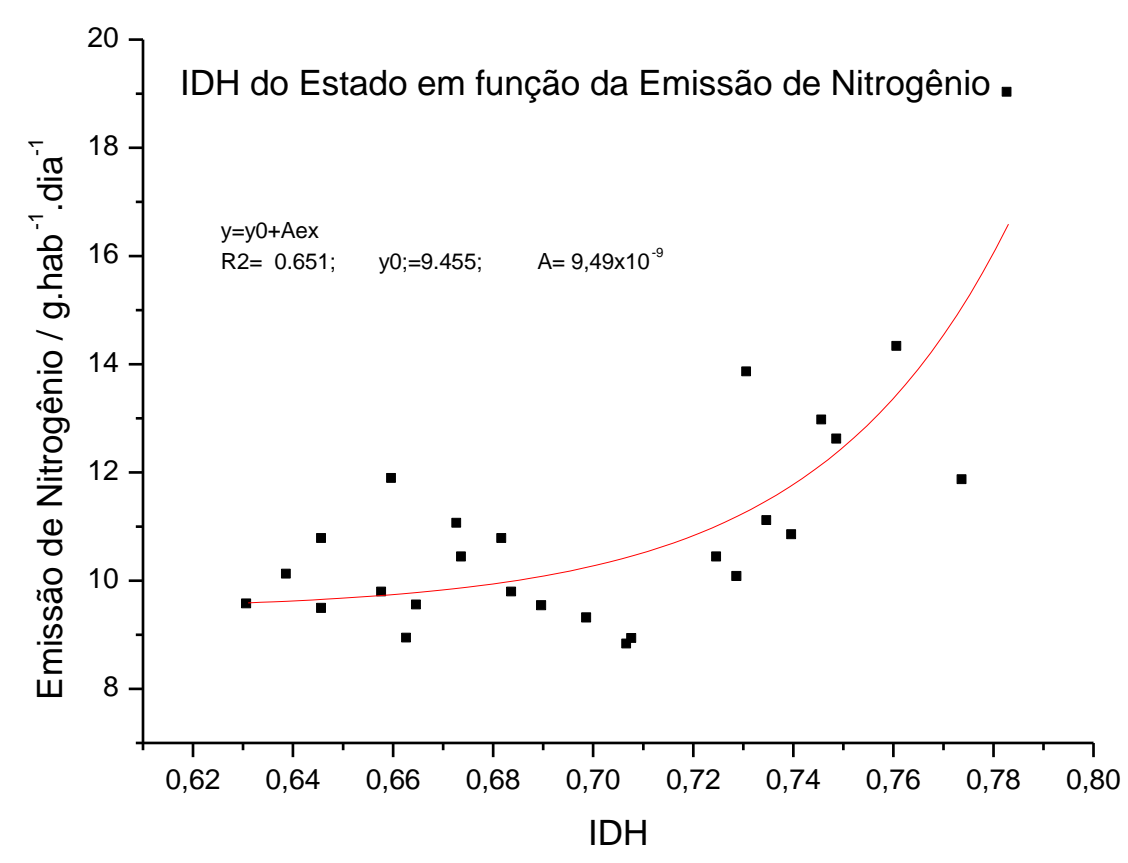

Figura 2 - Emissão de nitrogênio em função do IDH dos estados.

A emissão de nitrogênio também mostrou um aumento exponencial em função do IDH, importante ressaltar que na modelagem da emissão 0 aumento foi exponencial em função do PIB é logarítmico, o que mostra que no índice de IDH já está intrínseco fatores para normatizar esta grande variação no PIB. 
Com objetivo de padronizar o crescimento populacional, para verificar somente a contribuição do desenvolvimento econômico, na emissão de nitrogênio de cada estado foi construída a Figura 3 abaixo.

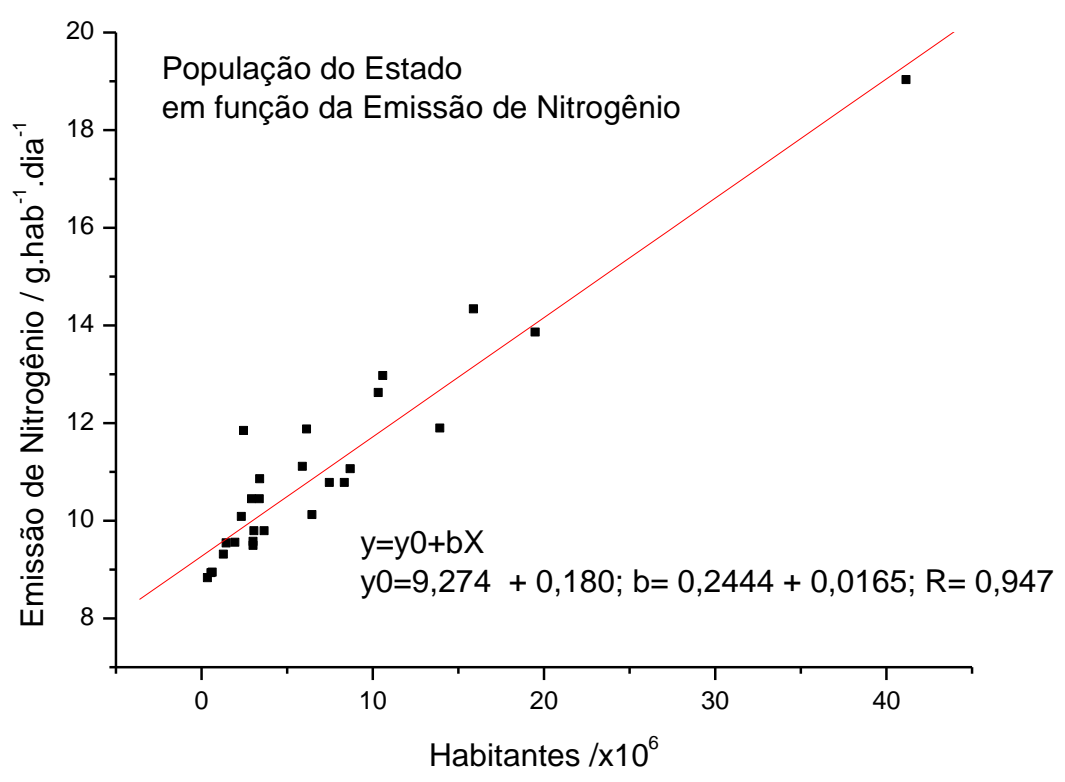

Figura 3 - Emissão de nitrogênio em função da população dos estados.

Verifica-se que a emissão de nitrogênio cresce linearmente com o aumento da população, comparando com os valores do IDH e PIB é possível verificar que o aumento destes índices tem como consequência uma aceleração na emissão de nitrogênio.

Estes dados mostram que o aumento nas emissões de nitrogênio é mais um fator que corrobora a premissa: quanto mais desenvolvido um país é, maior o impacto ambiental que ele causa e nosso papel é conhecer melhor este impacto com o objetivo de mitiga-lo ao máximo.

\section{CONCLUSÕES}

O desenvolvimento industrial e a modernização da atividade agrícola aumentaram as emissões de nitrogênio, alterando seu ciclo natural causando 
desequilíbrios ambientais. O nitrogênio é um elemento essencial à vida e a produção de alimentos, porém, em excesso traz problemas a saúde e ao meio ambiente.

No Brasil, o estado que mais libera nitrogênio na atmosfera é São Paulo, cerca de 11,3 gramas por pessoa por dia, devido a sua densidade populacional e por estádio de industrialização. O Brasil, por sua vez, emite 12,2 gramas por pessoa por dia, valor concordante com o encontrado na teoria. Na literatura tem-se que países mais desenvolvidos emitem de 15 a 18 gramas por pessoa por dia e os menos desenvolvidos 10 gramas (INTEGRATED MODELLING OF GLOBAL ENVIRONMENTAL, 2006, p.133).

Porém, o Brasil vem aumentando suas emissões ao longo dos anos, isso se dá pelo desenvolvimento industrial e a produção agrícola intensa. Com a modernização agrícola, houve um empobrecimento do solo, assim, cada vez mais usa-se fertilizantes para aumentar a produção e estes, por sua vez, contribuem significativamente com a quantidade de nitrogênio na atmosfera.

Diferentes índices de desenvolvimento, IDH e PIB, tem mostrado um aumento exponencial na emissão de nitrogênio, mostrando uma aceleração nesta emissão quando comparado com emissão por habitante

\section{6-REFERÊNCIAS}

BOBBINK, R; HORNUNG, M; ROELOFS, J.G.M. The effects o fair-borne nitrogen pollutants on species diversity in natural and semi-natural European vegetation. Journal of Ecology. Europa, v. 86, p. 717-738, 1998.

BORTOLI, M. et al. Emissão de óxido nitroso nos processos de remoção biológica de nitrogênio de efluentes. Engenharia Sanitária e Ambiental, Rio de Janeiro, v. 17, n. 1, 2012. Disponível em: <http://www.scielo.br/scielo.php?script=sci_arttext\&pid=S1413-41522012000100003>. Acesso em 07 abr. 2014.

BOUWMAN, A.F. et al. Global Inventory of Ammonia Emissions from Global Livestock Production and Fertilizer Use. Potomac, 2006.

BOUWMAN, A.F. et al. Nitrogen Surfaces balances in Intensive Agricultural production systems in diferente world regions. Pedosphere, Potomac, v. 15, n. 2, p. 137-155, 2005.

CÓNSUL, J.M.D. et al. Decomposição catalítica de óxidos de nitrogênio. Química Nova, São Paulo, v. 27, n. 3, 2004. Disponível em: <http://www.scielo.br/scielo.php?pid=S010040422004000300013\&script=sci_arttext>. Acesso em: 07 abr. 2014.

GALLOWAY, J.N. et al. Nitrogen cycles: Past, presente, and future. Biogeochemistry, v. 70, p. 153-226, 2004. 
INTEGRATED MODELING OF GLOBAL ENVIRONMENTAL CHANGE. Holanda: MNP, out. 2006. Nitrogênio é apontado como novo vilão do ecossistema. HYPESCIENCE. São Paulo, 2010. Disponível em:<http://hypescience.com/nitrogenio-e-apontado-como-novo-vilao-do-ecossistema/>. Acesso em: 07 abr. 2014.

NUVOLARI, Ariovaldo et al. Reúso da Água: conceitos, teorias e práticas. 01. ed. São Paulo: Blucher, 2007.

VAN DRECHT, G. et al. Global modeling of the fate of nitrogen from point and nonpoint sources in soils, groundwater and surfasse water. Global Biogeochemical Cycles., 2003. 